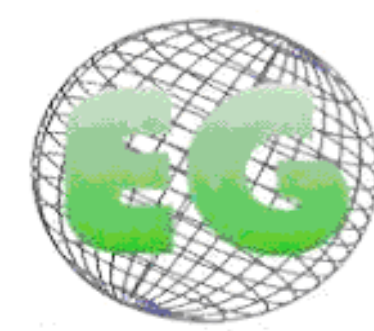

ISSN 1695-6141

$N^{\circ} 23$
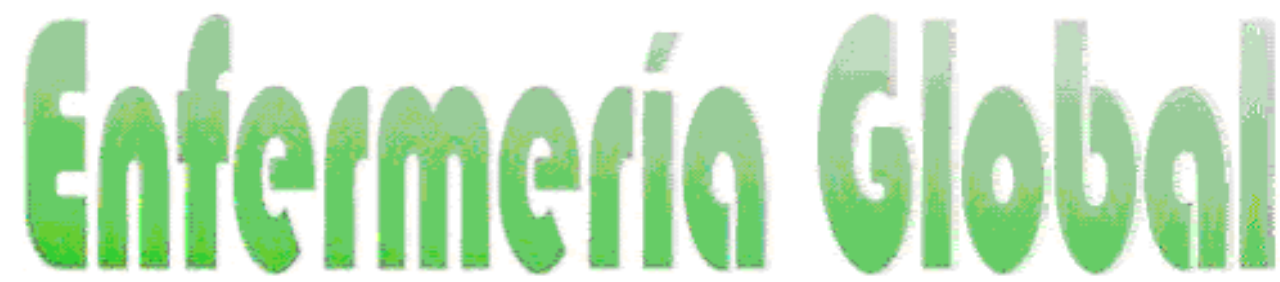

Revista electrónica trimestral de Enfermería

www.um.eslegloball

CLÍNICA

\title{
Cuidados de enfermería a pacientes con falla multiorgánica que reciben hemoderivados
}

Nursing care for patients with multiple organ failure receiving blood products

\author{
*Tovar, D., **Mejías, M., "Rodríguez, L. \\ *Profesor/a Asistente. "**Profesora Agregada. Universidad de Carabobo. Venezuela. \\ Palabras clave: Transfusiones sanguíneas; Cuidados de enfermería; Administración de hemoderivados. \\ Keywords: Blood transfusion; Nursing; Administration of blood products.
}

\section{RESUMEN}

La presente investigación comparó el conocimiento que poseen las enfermeras de la Emergencia de Adultos de la Ciudad Hospitalaria Dr. Enrique Tejera antes y después de participar en un programa educativo sobre los cuidados de enfermería a pacientes con falla multiorgánica que reciben hemoderivados referidos a: cuidados de enfermería antes, durante y después de la administración del mismo, a través de una muestra de 20 enfermeras de atención directa.

Esta investigación se desarrolló entre el año 2007 y 2008, en la ciudad de Valencia, Estado Carabobo, Venezuela. El diseño de la investigación corresponde al tipo cuasi-experimental con un solo grupo pre-test y post-test; la recolección de los datos se realizó mediante un cuestionario de 41 preguntas de opción múltiples; los resultados obtenidos demostraron que la aplicación del programa educativo incrementó significativamente $(p<0,001)$ el conocimiento de las enfermeras en relación a los cuidados de enfermería en la administración de hemoderivados a pacientes con falla multiorgánica referidos a: cuidados antes, durante y después de la administración de los mismos. Los resultados permiten concluir que la aplicación del programa educativo en las instituciones de salud, sobre la administración de hemoderivados, es beneficioso para proporcionar, reafirmar, y ampliar los conocimientos del personal de enfermería en servicio.

\section{ABSTRACT}

This study compared the knowledge of nurses at the Dr. Enrique Tejera Adult Emergency City Hospital before and after participating in an educational program on nursing care to patients with organ failure who receive blood products relating to: care nursing before, during and after administration, with a sample of 20 nurse practitioners. The research was performed between 2007 and 2008 in the city of Valencia, Carabobo, Venezuela. The research design was quasi-experimental for a single pre-test and post-test group. Data collection was through a questionnaire of 41 multiple choice questions and the results obtained showed that the implementation of the educational program increased significantly $(p<0.001)$ the knowledge of nurses regarding nursing care in the 
administration of blood products to patients with multiple organ failure in relation to care before, during and after administration of the same. The results conclude that the implementation of the educational program on the administration of blood products in health institutions is beneficial to provide, reaffirm and expand knowledge of nursing staff on duty.

\section{INTRODUCCIÓN}

La sangre ha sido considerada como un factor de salud y fuerza para el ser humano desde los tiempos de los orígenes del hombre, debido a que ella es la encargada de conectar entre sí todas las células del organismo con el fin de satisfacer las necesidades y/o requerimientos necesarios en los tejidos, para mantener el equilibrio del organismo. Dada la importancia de la sangre para la supervivencia humana, despertó en varios investigadores, entre ellos el fisiólogo Jean-Baptiste Denys en 1667, la necesidad de realizar las primeras transfusiones sanguíneas, con la particularidad que estas fueron realizadas de un animal a un ser humano, obteniéndose la mayoría de las veces, resultados fatales. Sin embargo, esto no evitó que otros investigadores siguieran realizando este tipo de experimentos, aunque el resultado fuese siempre el mismo, un desastre detrás de otro.

Estos resultados motivaron que esta práctica se abandonara por 150 años. Con el transcurrir de los años, Blundell en 1818 logró con éxito las primeras transfusiones entre humanos; sin embargo, la tendencia de la sangre a coagularse, lo rudimentario de los equipos de transfusión, y sobre todo la alta mortalidad inexplicable en aquel entonces, abrieron el camino para desarrollar más investigaciones que permitieran mejorar la realización de este procedimiento.

Estas investigaciones sirvieron de base para lo que hoy se conoce como transfusiones sanguíneas, cuya técnica consiste en administrar sangre o alguno de sus derivados, de un donante a un receptor. Cabe destacar que para aquella época este procedimiento pasó a ser considerado como difícil y peligroso para el receptor, producto del gran desconocimiento que se tenía sobre el mismo; pero fueron estos intentos fallidos los que motivaron las investigaciones al respecto, dando origen posteriormente a los conocimientos que hasta el presente son los pilares fundamentales en las transfusiones sanguíneas.

Estas investigaciones permitieron el nacimiento de la inmunología, la descripción de los antígenos de superficie $A B O$ de las células rojas, la creación de los bancos de sangre y el descubrimiento de la transmisión de enfermedades infecto-contagiosas a través de las transfusiones sanguíneas, lo que ocasionó la producción de nuevos conocimientos en la hemoterapia generando grandes avances científicos aunado a la demanda cada vez mayor de la población a las transfusiones sanguíneas debido a las diferentes patologías en las cuales estas constituyen la salvación o por lo menos el tratamiento de las mismas.

De igual manera, la globalización del conocimiento permitió el desarrollo de la hemoterapia y los bancos de sangre a nivel mundial. El auge de nuevos conocimientos ameritó la formación de personal calificado para el manejo, preparación y conservación de los hemoderivados; lo que permitió a enfermería formarse en este campo.

En el caso particular de Venezuela, desde 1942 se inició la preparación del personal de enfermería en hemoterapia con el fin de tener un personal académicamente capacitado, 
disciplinado, responsable y con mística de trabajo que se encargara del procesamiento de los hemoderivados en cuanto a producción, conservación y mantenimiento de los mismos ${ }^{(1)}$ De esta manera, se crea la especialidad en hemoterapia, la primera especialidad de enfermería en el país, la cual permitió garantizar personal de enfermería capacitado con habilidades y destrezas en el manejo, elaboración y conservación de estos productos. Es de hacer notar, que esta especialidad no era impartida directamente por las universidades conformadas en el país para el momento, sino que eran impartidas por los bancos de sangre de algunos hospitales del país, especialmente por aquellos ubicados en la ciudad de Caracas. Los profesionales de enfermería que egresan de este tipo de especialidades son formados para vincularse directamente con el hemoderivado, es decir, ellos realizan los procesos de extracción de sangre del donador, preparación del hemoderivado, pruebas serológicas y de compatibilidad, almacenamiento, y conservación de los mismos, mas no establecen una relación con el paciente que será el receptor del hemoderivado, debido a que estas especialidades no contemplan la formación del personal de enfermería que se encargue tanto de la preparación y administración del hemoderivado como del cuidado al paciente que lo recibe, trayendo como consecuencia que se produjese una ruptura en el proceso de cuidado que se le brinda al paciente antes, durante y después de la transfusión sanguínea.

Es importante destacar que en la actualidad el personal de Enfermería se encuentra carente de especialidades en hemoterapia avaladas por universidades reconocidas que se encarguen de capacitar universitariamente al personal de enfermería; no obstante, se cuenta con residencias asistenciales avaladas por los diferentes bancos de sangre de los hospitales del país, pero estas no satisfacen las necesidades que el área requiere, ya que es común observar un déficit importante de enfermeras hemoterapistas en todo el país. Asimismo estas residencias asistenciales generan una situación particular, debido a que se enfocan solo a la capacitación del personal de enfermería en el proceso de elaboración, manejo, y conservación del hemoderivado, obviando la necesidad de una formación integral del personal de enfermería, en cuanto al manejo del hemoderivado (producción, manejo, conservación y mantenimiento) y los cuidados de enfermería que debe recibir el paciente antes, durante y después de la administración del hemoderivado, es decir, una formación que permita a la enfermera desenvolverse en ambas fases del procedimiento, ya que tanto el manejo del hemoderivado como la administración del mismo son fundamentales para la vida y el cuidado del paciente.

Esta situación antes descrita ha sectorizado tanto el cuidado, ya que al presentarse un problema al momento de la transfusión sanguínea y solicitar las enfermeras de atención directa de los servicios la ayuda respectiva al personal de banco de sangre, la información proporcionada por ellos no satisface las dudas del personal de enfermería que administra el hemoderivado. En lo que respecta a este último punto, tal vez esa falta de énfasis en la preparación del personal que se encarga de la administración del hemoderivado deriva de lo expresado en la legislación venezolana en su artículo 20 sobre el uso de los hemoderivados que expresa: la transfusión de sangre es responsabilidad del médico, quien deberá vigilar al paciente el tiempo necesario y suficiente para prestar su oportuna asistencia en caso de que se produzcan reacciones; asimismo será responsable por las consecuencias patológicas que puedan desarrollarse posteriormente en el paciente derivados de la transfusión y que sean causadas por su omisión, impericia o negligencia ${ }^{(2)}$

En contraposición con lo anteriormente expuesto, en la práctica diaria es cotidiano observar que es el personal de enfermería quien se encarga de la administración y vigilancia tanto del paciente como del hemoderivado, y es en muchas ocasiones quien toma las primeras medidas ante cualquier complicación o reacción adversa que se pueda producir durante o 
después de la administración del hemoderivado, ya que por lo general no se cuenta siempre con la presencia de un médico en los servicios donde se están administrando los mismos. Entonces, si éste se ha convertido en un procedimiento básicamente del personal de enfermería, se requiere que el mismo posea un conocimiento profundo sobre el tema, un manejo meticuloso de la atención al paciente y la aplicación correcta de un protocolo, especialmente para prevenir las complicaciones que pueden presentarse en el paciente que recibe la transfusión.

Todos los puntos desarrollados anteriormente han hecho que se produzcan desatinos en los cuidados de enfermería que se les brindan a los pacientes antes, durante y después de la administración de hemoderivados, realizando en muchas oportunidades este procedimiento de una manera mecánica y como parte de una rutina más del servicio; trayendo como consecuencia fallas en la valoración física del paciente antes de la administración del hemoderivado, la cateterización o permeabilización con solución fisiológica de un acceso venoso de buen calibre antes de la llegada al servicio del hemoderivado que disminuya los retrasos en la administración y por consiguiente la exposición del hemoderivado a altas temperaturas que pongan en riesgo su conservación, la falta de explicación del procedimiento lleva implícito que se obvie la correspondiente firma del consentimiento informado por el paciente o familiares para autorizar la administración del hemoderivado, así mismo la administración de medicamentos previos a la transfusión ${ }^{(3)}$ que son en su mayoría indicados para evitar la aparición de reacciones adversas, en algunas oportunidades no es llevada a cabo.

Es importante saber que estos cuidados no implementados forman parte de algunos de los múltiples cuidados de enfermería que se deben llevar a cabo antes de la administración del hemoderivado según Cahill, este panorama anteriormente descrito desencadena un ciclo que se perpetúa en las siguientes fases del procedimiento, ya que no es extraño observar que una vez iniciada la transfusión sanguínea se obvie realizar el monitoreo de los signos vitales del paciente que indique su estado de salud durante el desarrollo del procedimiento, la velocidad de infusión del hemoderivado no es controlada, transcurriendo varias horas antes de que se termine la administración del mismo, lo que podría llevar a una contaminación bacteriana del hemoderivado debido a la tardanza en la infusión del mismo. En este mismo orden de ideas, una vez finalizada la transfusión, el paciente no es valorado para comprobar la estabilidad de su estado de salud, y mucho menos es educado sobre los síntomas de alarma que podría presentar, si tuviese una complicación derivada de la administración del hemoderivado, y se obvia realizar las anotaciones de enfermería respectivas que permitan llevar un record de todas aquellas transfusiones realizadas y que por alguna u otra razón desencadenaron o no un problema en el paciente.

De lo anteriormente expresado y de lo que sucede en el día a día en la práctica enfermera, se puede inferir que esos desatinos que se producen a lo largo del procedimiento son debidos en primer lugar a que la anamnesis del paciente antes del procedimiento se lleva a cabo con poca profundidad y por consiguiente esto no permite desarrollar entre el paciente y la enfermera esa relación de ayuda idónea para llevar a cabo el procedimiento, este factor trae como consecuencia que no se produzca el establecimiento de la relación enfermerapaciente, relación de ayuda que es el fundamento de la práctica enfermera clínica: el elemento esencial de cuidados con cada cliente, en cada situación ${ }^{(4)}$; en segundo lugar al realizar la revisión de las diversas investigaciones sobre el conocimiento que poseen las enfermeras sobre los cuidados de enfermería antes, durante y después de la administración de hemoderivados tenemos que la mayoría refleja que existe un porcentaje significativamente alto de enfermeras que no poseen el conocimiento para la realización del procedimiento $^{(5)}$ 
A través del presente estudio se planteó la necesidad de elevar la calidad de cuidado que se brinda en las instituciones de salud a través de la aplicación de un programa educativo que satisficiera las necesidades de conocimiento del personal que realiza la transfusión sanguínea.

\section{OBJETIVOS}

\section{General}

Comparar el conocimiento que poseen las enfermeras de la Emergencia de Adultos de la Ciudad Hospitalaria Dr. Enrique Tejera antes y después de participar en un programa educativo sobre los cuidados de enfermería a pacientes con falla multiorgánica que reciben hemoderivados referidos a: cuidados de enfermería antes, durante y después de la administración del mismo.

De este objetivo general se desprenden 03 objetivos específicos cada uno de los cuales dirigido a los factores objetos de estudio:

1.- Comparar el conocimiento que poseen las enfermeras de la Emergencia de Adultos de la Ciudad Hospitalaria Dr. Enrique Tejera antes y después de participar en un programa educativo sobre los cuidados de enfermería a pacientes con falla multiorgánica que reciben hemoderivados referidos a: cuidados de enfermería antes de la administración del mismo.

2.- Comparar el conocimiento que poseen las enfermeras de la Emergencia de Adultos de la Ciudad Hospitalaria Dr. Enrique Tejera antes y después de participar en un programa educativo sobre los cuidados de enfermería a pacientes con falla multiorgánica que reciben hemoderivados referidos a: cuidados de enfermería durante la administración del mismo.

3.- Comparar el conocimiento que poseen las enfermeras de la Emergencia de Adultos de la Ciudad Hospitalaria Dr. Enrique Tejera antes y después de participar en un programa educativo sobre los cuidados de enfermería a pacientes con falla multiorgánica que reciben hemoderivados referidos a: cuidados de enfermería después de la administración del mismo.

\section{MATERIALES Y METODO}

Esta investigación fue descriptiva, de tipo cuasiexperimental con un solo grupo pre-test y post-test de corte transversal, desarrollado en los meses de Enero - Marzo del año 2007 en la Ciudad Hospitalaria Dr. Enrique Tejera de la ciudad de Valencia - Edo. Carabobo Venezuela. La muestra estuvo conformada por 20 enfermeras de atención directa, quienes previa firma del consentimiento informado llenaron el instrumento (pre-test), este instrumento fue tipo cuestionario previamente diseñado, validado por expertos y sometido a una prueba piloto para determinar la confiablidad del mismo, este estuvo estructurado en 2 partes, la primera parte estuvo conformada por los datos demográficos y la segunda parte estuvo conformada por 41 preguntas de opciones múltiples y por medio de las cuales se determinó el conocimiento de las enfermeras sobre los cuidados de enfermería antes, durante y después de la administración de hemoderivados en paciente con falla multiorgánica.

Una vez recolectado el pre-test se procedió a desarrollar un programa educativo sobre los Cuidados de enfermería en la administración de hemoderivados en pacientes con falla multioganica, el mismo estuvo constituido por cuatro unidades las cuales tocaban los siguientes tópicos: 
UNIDAD I: Generalidades sobre los hemoderivados,

UNIDAD II: Cuidados de enfermería antes de la administración de hemoderivados,

UNIDAD III: Cuidados de enfermería durante de la administración de hemoderivados,

UNIDAD IV: Cuidados de enfermería después de la administración de hemoderivados.

Dicho programa se desarrolló en 2 sesiones educativas, de 2 horas cada una: una vez desarrollado el programa se procedió a recolectar la ultima parte de la información (Posttest), a todos estos datos se le procedió a realizar el análisis estadístico, para ello se utilizó el análisis de comparación de las puntuaciones usándose la t de Student para el análisis de medias de grupos pareados.

\section{RESULTADOS}

Una vez realizado el análisis estadístico de los datos se obtuvieron los siguientes resultados:

1.- De los 20 profesionales de enfermería estudiados, el $85 \%$ pertenecían al sexo femenino, asimismo tenían edades comprendidas entre los grupos etarios 20 a 24 años y entre 40 o más años con un 30\% para cada grupo etario, el $60 \%$ de los estudiados eran licenciados en enfermería, el 50\% tenía entre 5 a 10 años de servicio, asimismo el 50\% de los estudiados laboraban en el turno nocturno.

2.- En cuanto a los cuidados de enfermería antes de la administración de hemoderivados a pacientes con falla multiorgánica tenemos que el resultado obtenido por los participantes en la investigación, antes de la aplicación del programa educativo, fue de 14,5 puntos con desviación estándar de 3,4 puntos, mientras que después de haberlo aplicado, fue de 25,9 puntos con desviación de 1,1 puntos. La diferencia entre ambos valores que es de $+11,4$ fue significativa con un error menor del uno por mil $(p<0.001)$, dado el valor conseguido del estadístico de decisión t de Student que fue 15,761 para diez y nueve grados de libertad. Si se considera que el máximo posible a obtener era 27 puntos, el aumento de 11,4 puntos representa un incremento del $42,2 \%$ sobre el conocimiento de los participantes, este resultado implica que se produjo un incremento significativo sobre el conocimiento del factor evaluado (Gráfico $N^{\circ} 1$ ). 
GRAFICO No 6

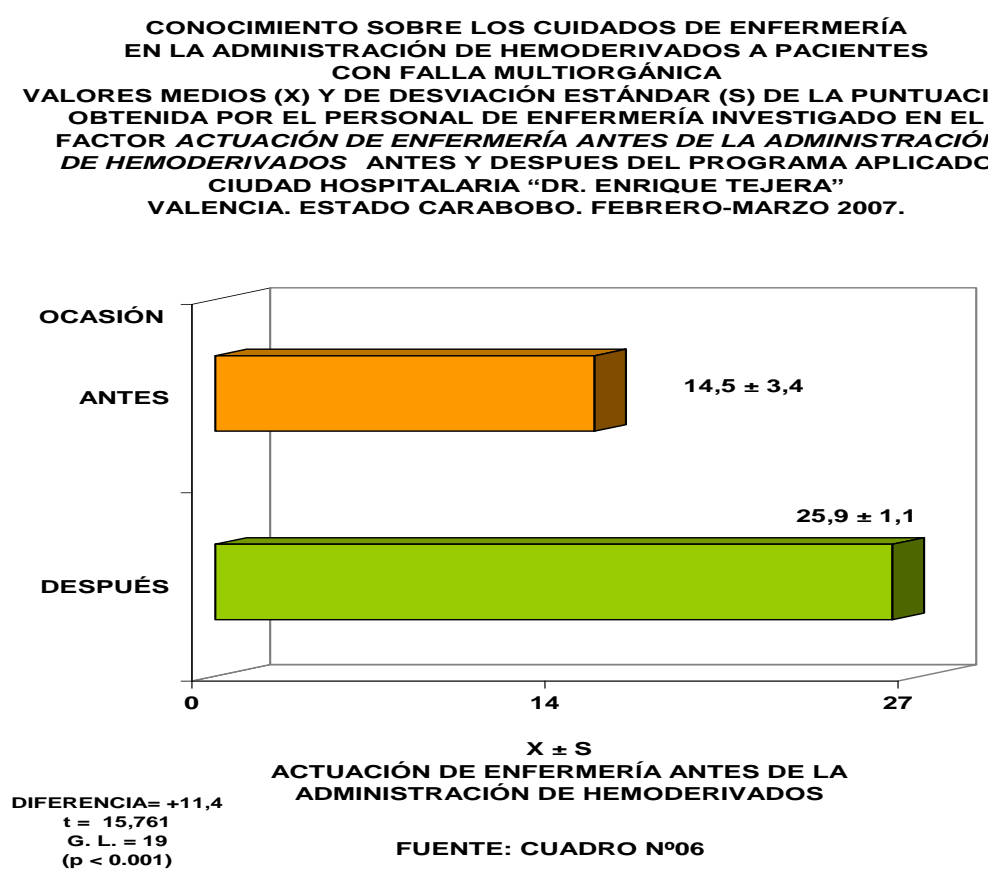

2.- En cuanto a los cuidados de enfermería durante la administración de hemoderivados a pacientes con falla multiorgánica tenemos que el resultado obtenido por los participantes en la investigación, antes de la aplicación del programa educativo, fue de 5,2 puntos con desviación estándar de 2,0 puntos, mientras que después de haberlo aplicado, fue de 10,1 puntos con desviación de 1,0 punto. La diferencia entre ambos valores, que es de $+4,9$ puntos, fue significativa con un error menor del uno por mil $(p<$ 0,001), dado que el valor conseguido del estadístico de decisión t de Student que fue 9,079 para diez y nueve grados de libertad. Si se considera que el máximo posible a obtener era 11 puntos, el aumento de 4,9 puntos representa un incremento del conocimiento en 44,5\%, este resultado implica que se produjo un incremento significativo sobre el conocimiento del factor evaluado (Gráfico №2). 


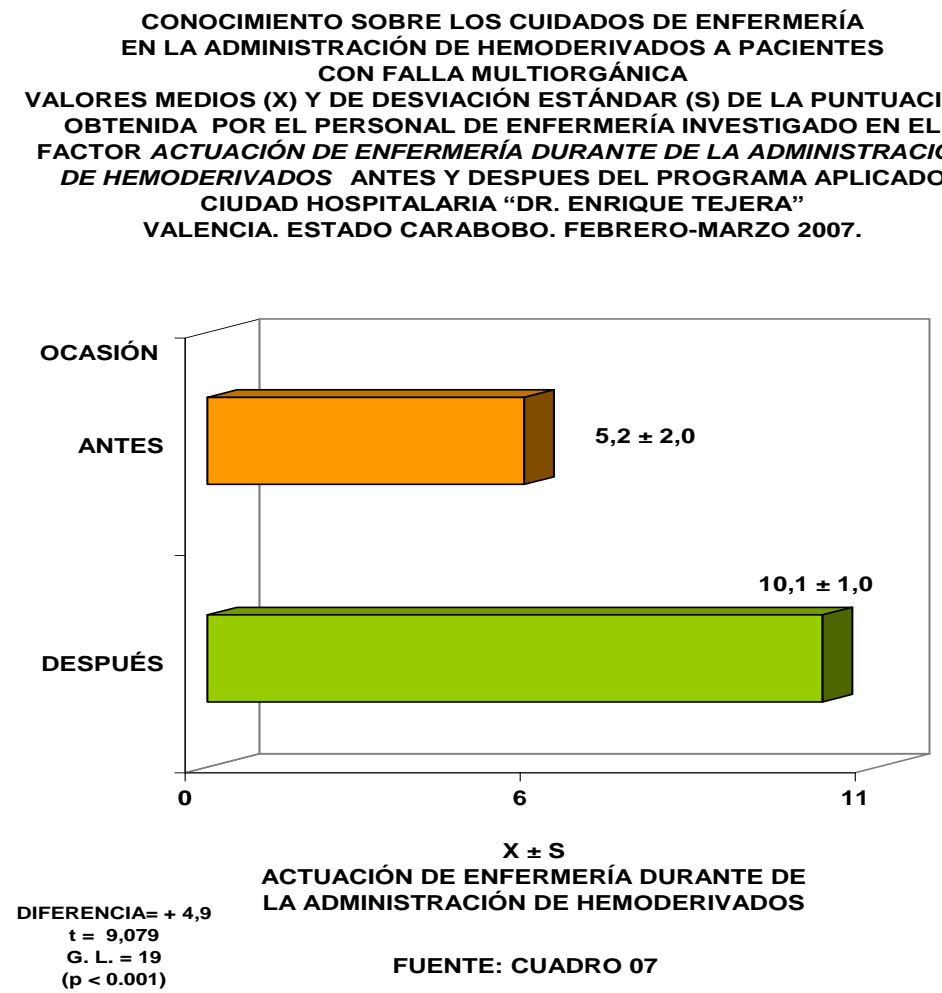

3.- En cuanto a los cuidados de enfermería después de la administración de hemoderivados a pacientes con falla multiorgánica tenemos que el resultado obtenido por los participantes en la investigación, antes de la aplicación del programa educativo, fue de 0,9 puntos con desviación estándar de 0,8 puntos, mientras que después de haberlo aplicado, fue de 2,7 puntos con desviación de 0,5 puntos. La diferencia entre ambos valores que es de 1,7 fue significativa con un error menor del uno por mil $(p<0.001)$, dado el valor conseguido del estadístico de decisión t de Student que fue 6,476 para diez y nueve grados de libertad. Si se considera que el máximo posible a obtener era 3 puntos, el aumento de 1,8 puntos representa un incremento en el conocimiento del 60,0\%, este resultado implica que se produjo un incremento significativo sobre el conocimiento del factor evaluado (Gráfico $\mathrm{N}^{\circ}$ $3)$. 


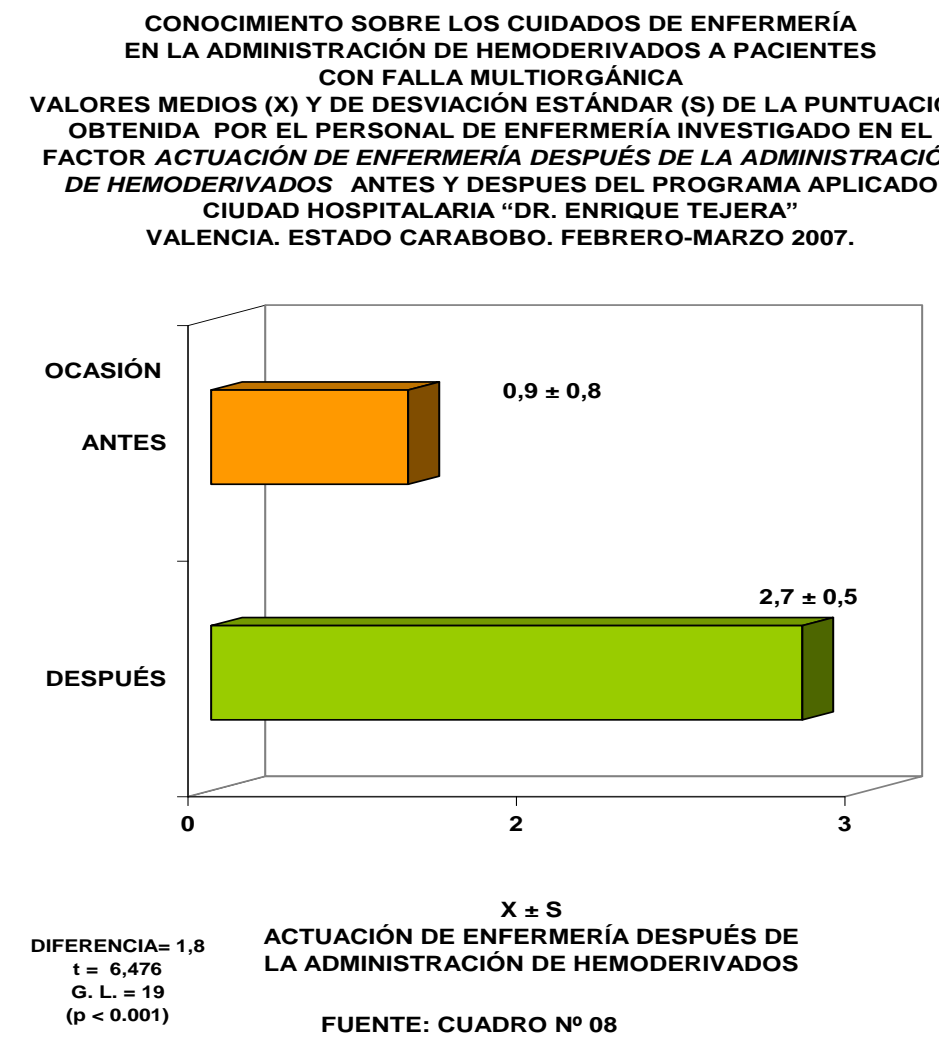

4.- En cuanto al nivel de conocimiento de los participantes antes y después de la aplicación del programa educativo sobre actuación de enfermería en la administración de hemoderivados a pacientes con fallas multiorgánica, tenemos que el resultado obtenido por los participantes en la investigación, antes de la aplicación del programa educativo, fue de 20,7 puntos con desviación estándar de 4,7 puntos, mientras que después de haberlo aplicado, fue de 38,8 puntos con desviación de 1,5 puntos. La diferencia entre ambos valores que es de $+18,1$ fue significativa con un error menor del uno por mil $(p<0.001)$, dado el valor conseguido del estadístico de decisión t de Student que fue 16,490 para diez y nueve grados de libertad. Si se considera que el máximo posible a obtener era 41 puntos, el aumento de 18,1 puntos representa un incremento del $44,1 \%$ sobre el conocimiento de los participantes, siendo por lo tanto efectivo el programa educativo aplicado en el personal de enfermería (Gráfico № 4). 


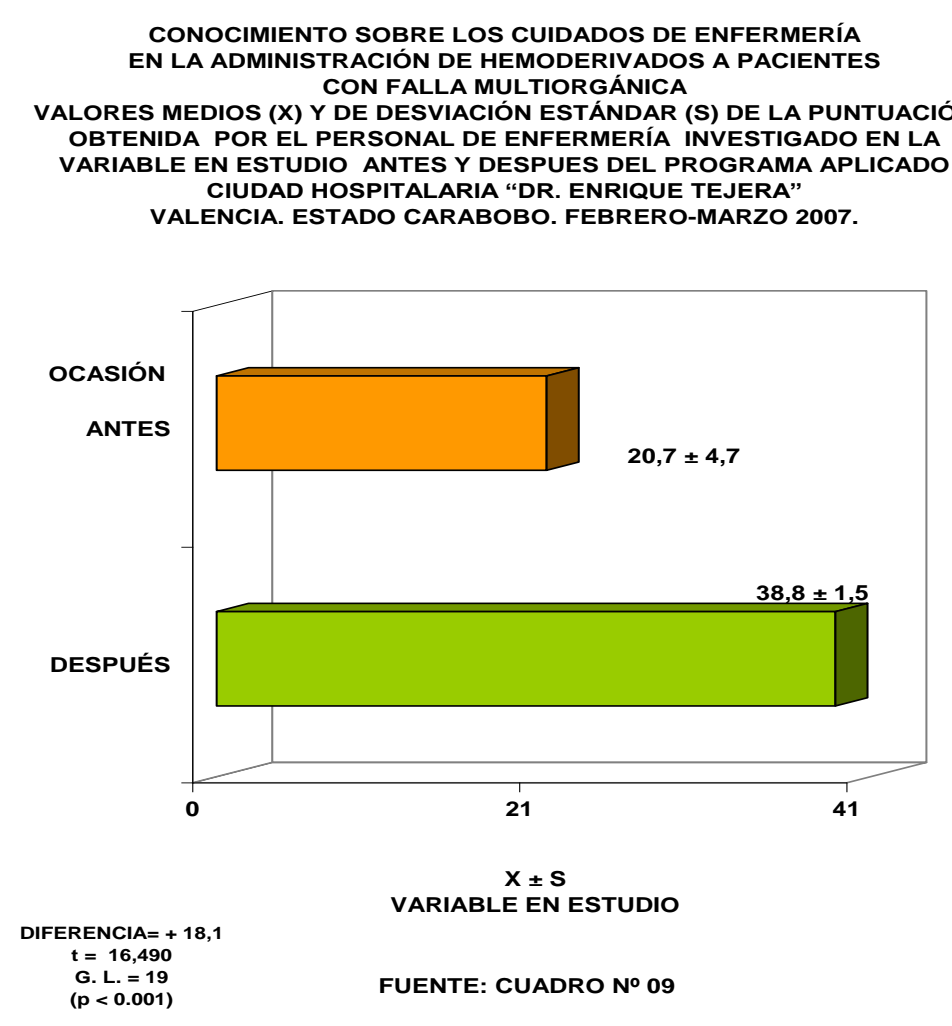

\section{DISCUSIÓN DE LOS RESULTADOS}

Las transfusiones sanguíneas son un procedimiento que cada día toma más relevancia en las instituciones de salud, debido al gran número de patologías a las que se encuentran relacionadas; como sabemos, cada año se realizan más y más campañas para incentivar la donación de sangre.

De forma global, el centro de interés de la disciplina enfermera indica aquello sobre lo que se orienta la practica enfermera, esta práctica se centra en el cuidado de la personas, se basa en brindar cuidados al paciente en todo momento, ya que el objetivo de los cuidados de enfermería consiste en proporcionarle al paciente cuidados individualizados, planificados y adecuados, en un entorno que favorezca el cuidado, el tratamiento, los objetivos de la rehabilitación y las necesidades especificas de cada cliente ${ }^{(6)}$ Es por ello que el proceso de cuidar implica una serie de fases como la reflexión, la integración de creencias y valores, el análisis crítico la aplicación de conocimientos, el juicio clínico y la intuición ${ }^{(7)}$, en concordancia con lo anteriormente descrito, la aplicación de conocimientos en la administración de hemoderivados es de vital importancia, ya que se requieren cuidados específicos en cada una de las fases de la administración (antes, durante y después).

Entre los cuidados antes de la administración de hemoderivados están los descritos por DI PASCUALE (8) quien expresa que el paciente debe estar informado del proceso al cual va a ser sometido y deberá avalar su decisión, suscribiendo el formato del consentimiento informado, así mismo debe tener la capacidad de entender lo que se le explica, contar con la información adecuada y suscribirlo sin ningún tipo de coerción. Por su parte, Merchan ${ }^{(9)}$ hace énfasis en que los cuidados durante la administración deben estar centrados en la velocidad del goteo, él indica que la infusión debe iniciarse a goteo lento durante los primeros 5 a 15 minutos, permaneciendo junto al paciente, de esta forma si el paciente 
muestra signos típicos de reacción adversa, interrumpir la transfusión, pero si al cabo de estos 15 minutos, no ha habido efectos adversos, es posible reevaluar los signos vitales del paciente e incrementar la velocidad de infusión .

Finalmente al culminar una transfusión sanguínea, los cuidados de enfermería a brindar en el paciente están dirigidos a detectar la presencia de reacciones agudas en él, esto se logra a través de la evaluación de los signos vitales del paciente de manera periódica, haciendo un registro de los mismos, esta valoración de los signos vitales debe realizarse una vez culminada la transfusión y hasta una hora después de culminada la administración del hemoderivado, además, el paciente debe estar en observación estrecha durante ese mismo periodo de tiempo, ya que las reacciones adversas, pueden presentarse durante la infusión o pocos minutos a horas después que se administró el hemoderivado, entre las reacciones más comunes tenemos: las alérgicas, febriles no hemolíticas, sépticas, hemolíticas, y la sobrecarga circulatoria. ${ }^{(10)}$

Estos resultados reafirman que la enfermera adquiere y desarrolla sin cesar los conocimientos a partir de un centro de interés que le es propio y de una concepción explicita de su disciplina (11) los conocimientos que adquiere sustentan sus acciones, y estos conocimientos le permiten priorizar y organizar sus actividades con el objetivo de proporcionar un servicio a la altura de la calidad, y es allí donde el desarrollo del programa educativo sobre la administración de hemoderivados permitió aumentar el conocimiento teórico y práctico de los profesionales que participaron en este estudio, logrando de esta manera: a) mantener a las enfermeras al día sobre las nuevas técnicas y conocimientos, b) ayudar a las enfermeras a adquirir destrezas en el área profesional especializada y c) transmitir a las enfermeras una información esencial para el desarrollo de la disciplina ${ }^{(12)}$; en otras palabras el desarrollo de este tipo de programa a través de la educación continua en servicio para el personal de enfermería es necesaria ya que permite mantener al personal de enfermería actualizado sobre los cuidados a brindar y al mismo tiempo disminuyen los riesgos y complicaciones que podrían sufrir un paciente.

\section{CONCLUSIÓN}

La enfermería como disciplina requiere y genera un cuerpo de conocimientos que sustentan su práctica diaria, éstos logran que el cuidado impartido sea de calidad y adaptado tanto a las necesidades del paciente como a las del campo laboral que requiere de sus servicios. Debido a que en la sociedad actual se produce una evolución constante del conocimiento humano, como consecuencia de las rápidas transformaciones que se llevan a cabo en la tecnología y la técnica, este crecimiento constante del conocimiento hace que la obsolescencia del mismo sea cada vez más acelerada y notoria, lo que requiere que el personal de enfermería se mantenga actualizado a través de programas educativos pertinentes los cuales juegan un papel fundamental en el desarrollo de la educación continua de los profesionales en servicio, convirtiéndose esta en una herramienta ideal para adaptarse a los cambios del mundo globalizado. Todo lo anteriormente expuesto y los datos obtenidos en la investigación, soportan la hipótesis de que los programas educativos aumentan significativamente el conocimiento de los profesionales de Enfermería que participan en ellos, y que los mismos deben implementarse en las instituciones de salud para mantener al personal actualizado. En el caso particular de esta investigación, el programa educativo aplicado estuvo relacionado con la administración de hemoderivados en pacientes con falla multiorgánicas, a través del cual se obtuvieron resultados significativos, pero cabe destacar que esta estrategia de actualización puede ser empleada a cualquier tema relacionado o no con la disciplina. 


\section{BIBLIOGRAFÍA}

1.- Revista de la Federación de Colegios de Enfermeras(os) Profesionales de Venezuela numero 01 Abril - Septiembre. Impreso por Publicidad Grafica Leon, S.R.L, 1994 2.- República Bolivariana de Venezuela Ley de Transfusión y Bancos de Sangre Gaceta, 1977. No. 31.356.

Disponible www.mijuicio.com/leyes/especiales/274.pdf

3.- Cahill, Mathew. Tratamiento en Enfermería. México. Editorial McGraw-Hill interamericana. Primera edición. 1991

4.- Potter, Patricia y Cols. Fundamentos de Enfermería. España. Editorial Harcourt/ Océano. Quinta edición. Volumen I, 2003

5.- Arias Karla y cols, Nivel de conocimiento del personal de enfermería en la administración de hemocomponentes en la Unidad de Emergencia Dr, Ruy Medina Morales del Hospital Central Universitario Antonio María Pineda de Barquisimeto - Estado Lara. Enero - Junio 2005. Disponible en : http://bibmed.ucla.edu.ve

6.- Martin Susan y cols . Normas de Cuidados del Paciente - Guía de Planificación de la Practica Asistencial Conjunta. Madrid - España. Editorial Harcourt/Brace. Sexta edición. 1997

7.- Kérouac Suzanne y Cols . El pensamiento Enfermero España. Editorial Elsevier Masson. Primera edición, 2007

8.- Di Pascuale, Stefania y col. Manual de Medicina Transfusional. México. Editorial McGraw-Hill interamericana. Primera edición. 2005

9.- Merchan, N. Protocolo De Atención a Paciente que Reciben Hemoderivados. 2006, Disponible en: http://www.uclm.es consultado en: 05/10/06

10.- Nettina, Sandra. Enfermería Practica de Lippincott. México. Editorial McGraw-Hill interamericana. Sexta edición. Volumen II. 2001

11.- Kérouac Suzanne y Cols. El pensamiento Enfermero. Ob. Cit. 2007

12.- Kozier, Bárbara. Fundamentos de Enfermería, Conceptos, Proceso y Practica. México.

Editorial McGraw-Hill interamericana. Quinta edición. Volumen I. 2002,

ISSN 1695-6141

@) COPYRIGHT Servicio de Publicaciones - Universidad de Murcia 